\title{
Label-free fluorescence predictions from large-scale correlative light and electron microscopy data
}

Ryan Lane ${ }^{1}$, Luuk Balkenende ${ }^{2}$, Simon van Staalduine ${ }^{2}$, Anouk Wolters ${ }^{3}$, Ben Giepmans ${ }^{4}$, Lennard Voortman ${ }^{5}$ and Jacob Hoogenboom ${ }^{1}$

${ }^{1}$ Technical University Delft, United States, ${ }^{2}$ Technical University Delft, Netherlands, ${ }^{3}$ University Medical Center Groningen, United States, ${ }^{4}$ University Medical Center Groningen, Groningen, Netherlands, ${ }^{5}$ Leiden University Medical Center, Netherlands

Volume electron microscopy (EM) has revolutionized the way in which biologists understand intra- and intercellular systems. Due to the lack of biological specificity, however, interpretation of the data often requires tedious expert analysis and annotation. For this reason fluorescence microscopy (FM) is often used in conjunction with EM, complementing structural data with targeted biological labels. The downside, however is that sample preparation protocols are often laborious, time-consuming, and potentially damaging to the sample. Correlating these multi-modal datasets presents another challenge, which is compounded when registering across large spatial extents and then again for 3D.

Deep convolutional neural networks (CNNs) are capable of inferring complex, non-linear relationships between images, simultaneously revolutionizing fields involved in image recognition tasks. Thus, we explored the potential for a CNN to make label-free fluorescence predictions from EM images. Inspired by the U-Net architecture [1, 2], the $\mathrm{CNN}$ is comprised of a multi-stage contraction path followed by a relatively shorter expansion path. This asymmetry is a result of the resolution difference between EM and FM images. The network is trained on largescale (several GBs) correlative FM and EM datasets amassed via an integrated array tomography workflow-serial section imaging facilitated by the use of an integrated fluorescence and electron microscope [3, 4]. Here we present fluorescence predictions on thin sections of rat pancreas tissue. Insulin-producing beta cells within the islet of Langerhans were immunolabelled with Alexa 594 and given a Hoechst counterstain.

Network predictions of Hoechst show high fidelity with the true fluorescent signal (Figure 1). Cell nuclei appear to be more easily discernible in the predicted image (Figure 1A) in part due to more uniform fluorescence throughout the nuclei as well as a reduced background. The correlation between the predicted and true fluorescence can be visualized by superimposing the signals onto the EM data (Figure 1C). Network predictions for the insulinproducing beta cells also show high qualitative agreement (Figure 2). While the network is capable of distinguishing different types of granules (Figure 2D), some clusters of insulin granules are predicted with noticeably low confidence (Figure 2E).

Network predictions are quantitatively evaluated based on the Pearson correlation coefficient (PCC): 0.511 for the nuclei-based dataset (Figure 1), and 0.765 for the insulin-based dataset (Figure 2). While the PCC serves as a reasonable indication of correlation, it has certain drawbacks stemming from practical limitations of the true fluorescence data. Inaccuracies in the multi-modal registration, aspecific labelling (Figure 1E), and bleed-through (Figure 1F) all result in spurious reductions to the PCC. Future work will be aimed at applying the CNN to other cell types and targets as well as to larger volume datasets. Early results indicate that network predictions may also offer benefits to organelle segmentation when used in conjunction with true FM and EM data.

\section{References}

[1] Ronneberger, O., Fischer, P., \& Brox, T. (2015, October). U-net: Convolutional networks for biomedical image segmentation. In International Conference on Medical image computing and computer-assisted intervention (pp. 234-241). Springer, Cham.

[2] Ounkomol, C., Seshamani, S., Maleckar, M. M., Collman, F., \& Johnson, G. R. (2018). Label-free prediction of three-dimensional fluorescence images from transmitted-light microscopy. Nature 

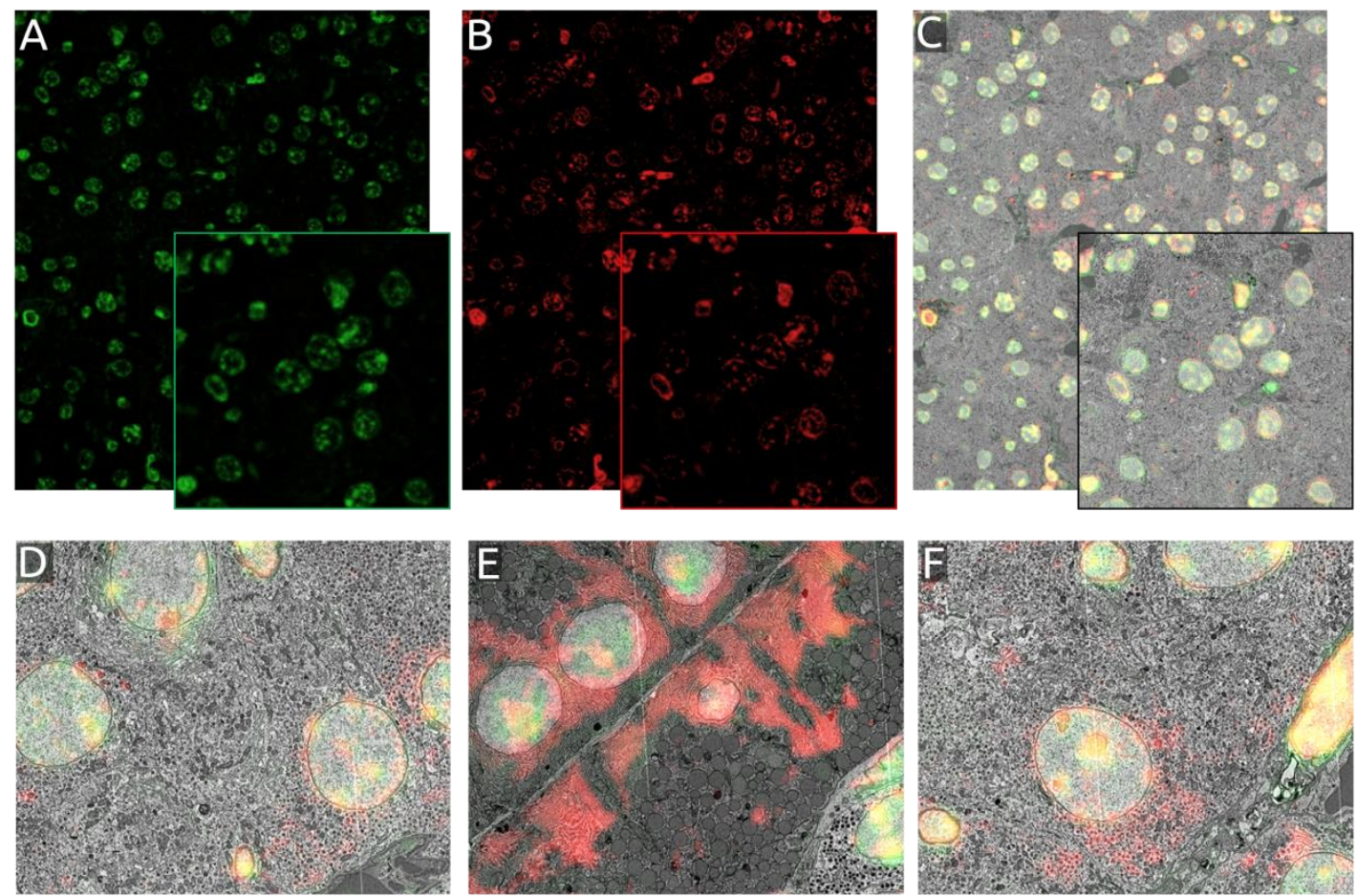

Figure 1. Figure 1. Network prediction (A) and true fluorescence signal (B) of Hoechst expression on a thin section of rat pancreas tissue. The true (red) and predicted (green) fluorescence overlaid on the EM data (C) with zoomedin regions of interest (D-F). (D) Zoomed-in region showing a relatively high degree of correlation between true and predicted fluorescence for cell nuclei. (E) Hoechst binds to large clumps of RNA in the exocrine region which the network omits. (F) Bleed-through of Alexa 594 fluorescence from insulin granules to the Hoechst channel.
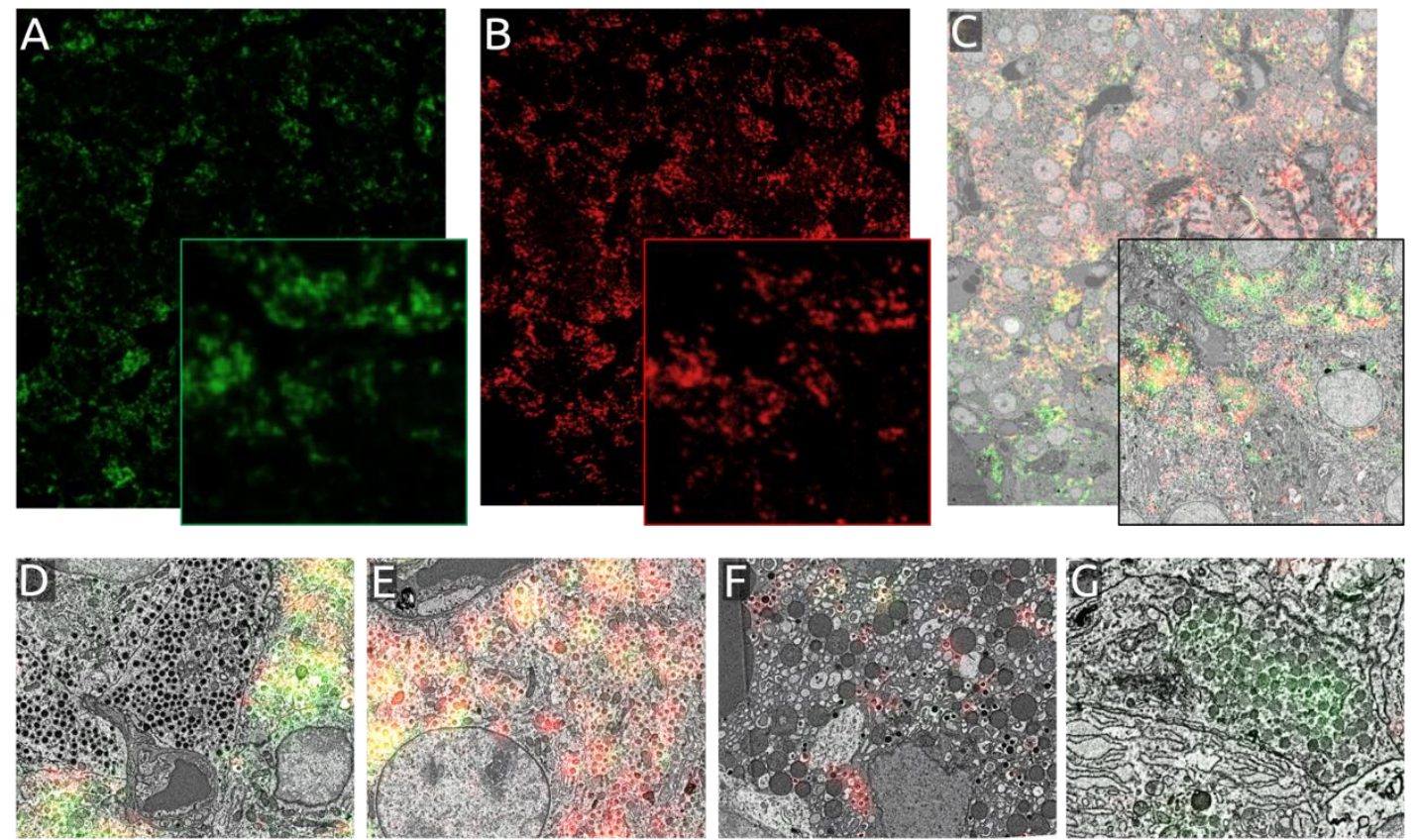

Figure 2. Figure 2. Network prediction (A) and true fluorescence signal (B) of Alexa 594 expression on a thin section of rat pancreas tissue. The true (red) and predicted (green) fluorescence overlaid on the EM data (C) with zoomed-in regions of interest (D-G). (D) Clusters of insulin granules exhibit a relatively high degree of correlation, while neighboring glucagon granules are correctly ignored. (E) A cluster of insulin granules for which the 
fluorescence is predicted with low probability. (F) Insulin granules camouflaged in a setting unfamiliar to the network. $(\mathrm{G})$ A spurious prediction for the presence of insulin granules.

\section{References}

[1] Ronneberger, O., Fischer, P., \& Brox, T. (2015, October). U-net: Convolutional networks for biomedical image segmentation. In International Conference on Medical image computing and computer-assisted intervention (pp. 234-241). Springer, Cham.

[2] Ounkomol, C., Seshamani, S., Maleckar, M. M., Collman, F., \& Johnson, G. R. (2018). Label-free prediction of three-dimensional fluorescence images from transmitted-light microscopy. Nature methods, 15(11), 917-920.

[3] Liv, N., Zonnevylle, A. C., Narvaez, A. C., Effting, A. P., Voorneveld, P. W., Lucas, M. S., ... \& Hoogenboom, J. P. (2013). Simultaneous correlative scanning electron and high-NA fluorescence microscopy. PloS one, 8(2), e55707.

[4] Lane, R., Vos, Y., Wolters, A. H., van Kessel, L., Giepmans, B. N., \& Hoogenboom, J. P. (2020). Optimization of negative stage bias potential for faster imaging in large-scale electron microscopy. Journal of Structural Biology: $X, 100046$. 